\section{Regenerative Medicine Blueprint}

\author{
Andre Terzic,, C. Michel Harper Jr., Gregory J. Gores, and Michael A. Pfenning
}

Regenerative medicine, a paragon of future healthcare, holds unprecedented potential in extending the reach of treatment modalities for individuals across diseases and lifespan. Emerging regenerative technologies, focused on structural repair and functional restoration, signal a radical transformation in medical and surgical practice. Regenerative medicine is poised to provide innovative solutions in addressing major unmet needs for patients, ranging from congenital disease and trauma to degenerative conditions. Realization of the regenerative model of care predicates a stringent interdisciplinary paradigm that will drive validated science into standardized clinical options. Designed as a catalyst in advancing rigorous new knowledge on disease causes and cures into informed delivery of quality care, the Mayo Clinic regenerative medicine blueprint offers a patient-centered, teambased strategy that optimizes the discovery-translation-application roadmap for the express purpose of science-supported practice advancement.

\section{INTRODUCTION}

Regenerative medicine offers a portfolio of technologies equipped to boost the innate healing potential of an individual, ensure repair of disease-afflicted tissues, and restore normative function [1]. Such innovation in the medical and surgical toolkit is poised to afford definitive solutions for a spectrum of disease conditions often limited today to recurring palliative care. Beyond the promise of substantive health benefits, disruptive regenerative technologies offer a radical approach to confronting inefficient treatments and es-

Center for Regenerative Medicine, Mayo Clinic, Rochester, Minnesota.

*(Correspondence : terzic.andre@mayo.edu) calating healthcare costs [2]. Curative options are viewed paramount in the context of changing pathodemographics that underscore an increased survivorship from acute and congenital diseases, yet an exponential aging of a global population ever more vulnerable to chronic, debilitating conditions [3].

Complementing current standard of care, nascent regenerative approaches progressively permeate in daily patient management, although the true value of such novel technologies remains to be firmly established. Technological breakthroughs have been fueled by advances in discovery science, exemplified by remarkable

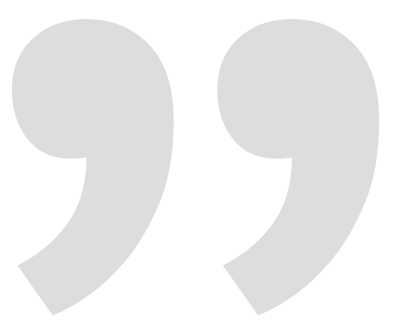

Beyond the promise of substantive health benefits, disruptive regenerative technologies offer a radical approach to confronting inefficient treatments and escalating healthcare costs.

progress in developmental and stem cell biology, tissue engineering, and systems informatics in this postgenomic era [4]. Broadly popularized, a growing number of clinical offerings have led to a heightened interest for regenerative medicine services by patients, their families, and more broadly by society at large. To address the expanding need, healthcare organizations - domestic and international-are designing and deploying dedicated regenerative medicine clinics, centers, and institutes aimed to facilitate the adoption and implementation of regenerative algorithms while ensuring the convergence of best practices across medical and surgical specialties [5]. 
Indeed, healthcare has an obligation to patients and society to move prudently, adhering to stringent safety/efficacy standards and rigorous ethical criteria, in order to rationally convert this promising science into proven therapies.

\section{Mayo Clinic regenerative medicine blueprint}

Mayo Clinic has embraced regenerative medicine as a strategic investment in the future of healthcare. The institutional strategy is envisioned as a science-supported practice advancement priority. It is executed through a discovery-translationapplication mandate, deployed across the organization. The evolving model of regenerative medicine encompasses the discovery, development, and delivery of next-generation patient management modalities targeted to address the root cause of disease and to offer the prospect of cures.

To this end, the Mayo Clinic Center for Regenerative Medicine has been established as a catalyst to advance newfound knowledge on disease causes and cures into informed delivery of effective quality care. The declared aims are to (i) discover, translate, and apply regenerative biomedical science into innovative clinical practice; (ii) develop regenerative medicine multidisciplinary team-centered programs, infrastructure, and workforce; (iii) advance regenerative medicine products and service lines; (iv) educate nextgeneration healthcare scientists and providers; (v) promote regenerative medicine global alliances and community partnerships; and (vi) establish regenerative medicine and surgery models of care.

To date, the Mayo Clinic Center for Regenerative Medicine has advanced efforts across multiple specialties, illustrating the diverse scope and depth of emerging strategies toward practice standardization and implementation. These extend from bone marrow transplantation in hematological malignancies and islet

\section{Mayo Clinic Regenerative Medicine Model}

- Strategic investment in the future of healthcare

- Institutional strategy envisioned as a science-supported practice advancement priority

- Executed through a discovery-translation-application mandate

- Deployed across the organization

- Encompasses discovery, development and delivery of patient management modalities

- Catalyst to advance new knowledge into informed delivery of quality care

The Mayo Clinic Center for Regenerative Medicine has advanced efforts across specialties towards practice standardization and implementation. The declared aims are to:

$>$ discover, translate and apply innovative science into clinical practice

$>$ develop team-centered programs, infrastructure and workforce

$>$ advance regenerative medicine products and service lines

$>$ educate next generation healthcare scientists and providers

$>$ promote global alliances and community partnerships

$>$ establish regenerative medicine and surgery models of care

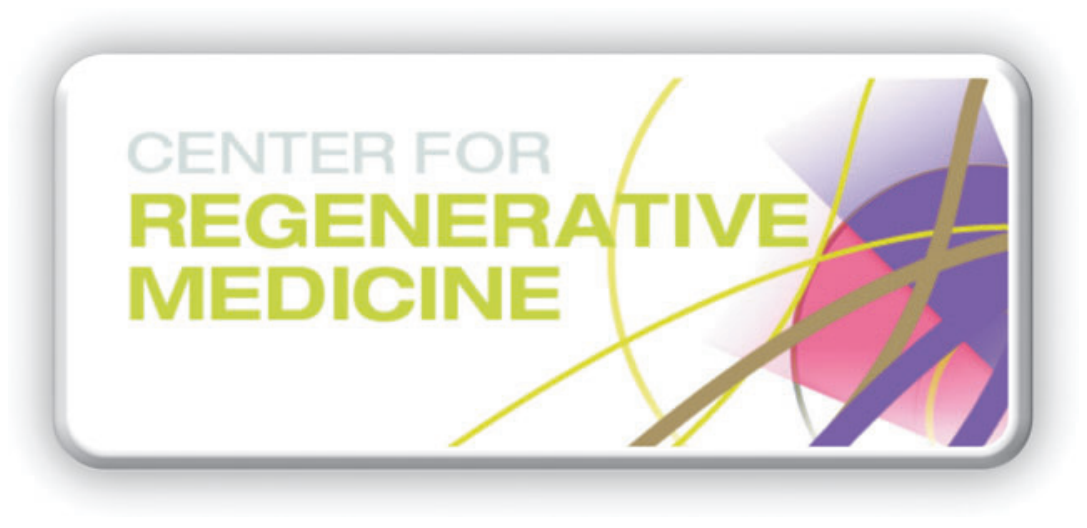

transplants for diabetes to the use of proregenerative adjuvants in wound healing; nerve reconstruction; facial reanimation and composite allotransplants in plastic and microsurgery; hybrid core decompression and osteochondral grafts in orthopedics; platelet-rich plasma interventions in sports medicine; cell therapies for neurodegenerative, cardiac, liver, and kidney disease; bionic regeneration in otherwise untreatable tracheal and laryngeal pathologies; or assist devices in organ failure management [6-18]. Clinical trials in regenerative medicine/surgery that are currently ongoing at Mayo Clinic and/or test Mayo Clinic-based technologies include studies of mesenchymal stem cells in amyotrophic lateral sclerosis and multiple systems atrophy; mononuclear cell therapy for hypoplastic left heart syndrome; $\mathrm{CD} 34^{+}$cells for acute myocardial infarction and refractory angina; lineage-specified cardiopoietic stem cells for chronic heart failure; stem cells for bronchiolitis obliterans, renal artery stenosis, aseptic necrosis of the hip, and osteoarthritis of the knee; stem cellcoated fistula plugs for Crohn's disease; stromal cells for host versus graft disease; and stem cells for relapsed ovarian 
cancer underscoring a broad range of multi-/interspecialty testing.

At a more fundamental level, advances have been made in decoding the determinants of cell fate and organogenesis; in the understanding of native processes for tissue renewal and organ rejuvenation; in the derivation of patient-/disease-specific biologics for safe and effective applications; and in the imaging and engineering of regenerative products [19-30]. Multimodal regenerative approaches are being refined to incorporate transplant of healthy tissues into damaged environments, prompt the body to enact a regenerative response in damaged tissues, and use tissue engineering to manufacture new tissue [31,32]. The Mayo Clinic's regenerative armamentarium encompasses cell-based biotherapies, tissue engineering technologies, and reparative platforms or, alternatively, use of devices to fortify failing organ function as a bridge to more definitive solutions. Emphasis is placed on optimization and standardization of procedures to ensure an upgraded regenerative response [33]. Beyond new therapies, diagnostic tools to follow individual regenerative capacity and to potentially serve as prognostic markers in disease management are also being developed [34,35].

The Mayo Clinic comprehensive framework - the "regenerative medicine blueprint"-is centered on the patient experience. The blueprint incorporates converging services encompassing the spectrum of regenerative care including dedicated work-ups, patient-specific biorepositories, and tailored procedures.

\section{Regenerative medicine work-up}

The concept of a "regenerative medicine clinic" has been advanced as a portal to educate people on existing regenerative services, to offer subspecialty referral, to enable collection of biospecimens, and to facilitate enrollment into clinical protocols [36]. Mayo Clinic has launched a consult service for regenerative medicine set as a singular point of access. The Mayo Clinic Regenerative Medicine Consult Service, harbored within the Transplant Center, provides medical evaluations and regenerative medicine work-ups for patients in whom the question has been raised whether a regenerative application is appropriate [1]. The consult service is designed to provide expert opinion on the risks/benefits of a regenerative approach and to address the value of available products or services across specialties and age groups. Systems and procedures in place enable the regenerative medicine consult service to participate in ongoing care plans and to be offered as an e-consult option.

\section{Regenerative medicine biorepository}

The regenerative medicine patient experience further integrates collection, banking, and processing of research and clinical-grade biospecimens for diagnostic and therapeutic needs. These needs range from diagnostic disease modeling, target identification/validation, and molecular screening to tailored therapeutic applications. The utility of patient-derived biologics in advancing regenerative diagnostic and therapeutic applications requires the preservation and maintenance of cell viability, best accomplished in a highly standardized and centralized environment [1]. Such is the rationale for the Mayo Clinic Regenerative Medicine Biotrust, a patient-derived resource for regenerative theranostics and the foundation for regenerative applications [37]. The biotrust functions ultimately to distribute regenerative products. This is accomplished through regulatory-compliant and integrated processes that encompass patient enrollment, sample collection and annotation, specimen profiling, and validation. Examples include umbilical cord blood banking under good manufacturing practice guidelines and the refinement of patient-specific pluripotent/lineagecommitted cytotypes across disease entities. A regenerative medicine biotrust provides a centralized resource that, in time, may advance into a lifelong disease risk managed personalized bioinsurance.

\section{Regenerative medicine procedures}

Today, most regenerative offerings target "no option" patients, that is, patients with advanced disease with a dismal prognosis. Increasingly, regenerative procedures are developed to cover preemptive interventions in early-stage disease or for disease prevention. Hence, the value of regenerative procedures or service lines must be demonstrated in the clinical and surgical practice relative to current accepted standards of care $[1,2]$. The delivery of regenerative service lines in either the medical or surgical setting depends critically on patient selection and disease stratification. To ensure optimal patient management, regenerative medicine service lines are developed with, and embedded within, existing medical specialties - regenerative medicine at point of care.

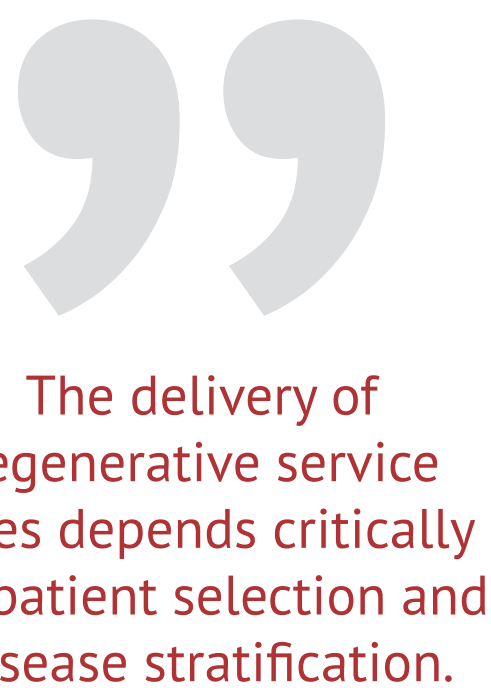

This point-of-care approach to advancing regenerative medicine at Mayo Clinic builds on an evolving model that presently consists of intersecting therapeutic strategies to disease management: 
- Prophylactic therapy-offsets side effects of standard of care therapy

- Combination therapy-augments efficacy of primary therapy

- Bridge therapy-delays transplantation in end-stage organ failure

- New organ therapy - offers fit-for-purpose solutions in "no option" patients

In response to patient needs, regenerative medicine at Mayo Clinic is moving forward with a knowledge-to-delivery paradigm [38] to advance regenerative science into new services and procedures that will steadily transform healthcare in providing definitive and affordable solutions. To ensure early and proper adoption, the rigor of comparative effectiveness analytics is needed to empower the incorporation of regenerative strategies into mainstream general practices.

\section{CONCLUSION}

An emergent core component of 21stcentury medicine and surgery, regenerative medicine, is positioned to transform healthcare in defining outcomes tailored to individual patient needs. Harnessing the prospect of regenerative medicine is predicated on a rigorous evidencebased approach. The Mayo Clinic regenerative medicine blueprint offers a paradigm of a patient-centric strategy, aimed to optimize the discovery-translation-application roadmap for the express purpose of advancing the science of regenerative medicine into innovative clinical practice.

\section{ACKNOWLEDGMENTS}

AT is Michael S. and Mary Sue Shannon Family Director, Center for Regenerative Medicine, and Marriott Family Professor of Cardiovascular Diseases Research. $\mathrm{CMH} \mathrm{Jr}$ is Executive Dean for Practice and Professor of Neurology. GJG is Kinney Executive Dean for Research and Reuben R. Eisenberg Professor of Medicine and Physiology. MAP is Deputy Director Administration, Center for Regenerative Medicine, Mayo Clinic.
Author disclosure

\section{statement}

The authors declare no potential conflicts of interest.

\section{REFERENCES}

1. Terzic A and TJ Nelson. (2013). Regenerative medicine primer. Mayo Clin Proc 88:766-775.

2. Terzic A and TJ Nelson. (2010). Regenerative medicine: advancing health care 2020. J Am Coll Cardiol 55:2254-2257.

3. Terzic A and SA Waldman. (2011). Chronic diseases: the emerging pandemic. Clin Transl Sci 4:225-226.

4. Terzic A, CD Folmes, A Martinez-Fernandez and A Behfar. (2011). Regenerative medicine: on the vanguard of health care. Mayo Clin Proc 86:600-602.

5. Matteson EL and A Terzic. (2013). Introduction to the symposium on regenerative medicine. Mayo Clin Proc 88:645-646.

6. Gertz MA, CB Reeder, RA Kyle and SM Ansell. (2012). Stem cell transplant for Waldenström macroglobulinemia: an underutilized technique. Bone Marrow Transplant 47:1147-1153.

7. Barton FB, MR Rickels, R Alejandro, BJ Hering, S Wease, B Naziruddin, J Oberholzer, JS Odorico, MR Garfinkel et al. (2012). Improvement in outcomes of clinical islet transplantation: 1999-2010. Diabetes Care 35:1436-1445.

8. Wong VW, GC Gurtner and MT Longaker. (2013). Wound healing: a paradigm for regeneration. Mayo Clin Proc 88:1022-1031.

9. Chen BK, AM Knight, GC de Ruiter, RJ Spinner, MJ Yaszemski, BL Currier and AJ Windebank. (2009). Axon regeneration through scaffold into distal spinal cord after transection. J Neurotrauma 26:1759-1771.

10. Amer H, BT Carlsen, JL Dusso, BS Edwards and SL Moran. (2011). Hand transplantation. Minn Med 94:40-43.

11. Martin JR, MT Houdek and RJ Sierra. (2013). Use of concentrated bone marrow aspirate and platelet rich plasma during minimally invasive decompression of the femoral head in the treatment of osteonecrosis. Croat Med J 54:219-224.

12. Finnoff JT, SP Fowler, JK Lai, PJ Santrach, EA Willis, YA Sayeed and J Smith. (2011). Treatment of chronic tendinopathy with ultrasound-guided needle tenotomy and platelet-rich plasma injection. PM R 3:900-911.

13. Ess KC. (2013). Patient heal thyself: modeling and treating neurological disorders using patient-derived stem cells. Exp Biol Med 238:308-314.

14. Bartunek J, M Vanderheyden, J Hill and A Terzic. (2010). Cells as biologics for cardiac repair in ischaemic heart failure. Heart 96:792-800.

15. Yu Y, JE Fisher, JB Lillegard, B Rodysill, B Amiot and SL Nyberg. (2012). Cell therapies for liver diseases. Liver Transpl 18:9-21.

16. Textor SC and LO Lerman (2013). Renal artery stenosis: medical versus interventional therapy. Curr Cardiol Rep 15:409.

17. Badylak SF, DJ Weiss, A Caplan and P Macchiarini. (2012). Engineered whole organs and complex tissues. Lancet 379:943-952.

18. Park SJ, SS Kushwaha and CG McGregor. (2012). State-of-the-art implantable cardiac assist device therapy for heart failure: bridge to transplant and destination therapy. Clin Pharmacol Ther 91:94-100.

19. van Wijnen AJ, J van de Peppel, JP van Leeuwen, JB Lian, GS Stein, JJ Westendorf, MJ Oursler, HJ Im, H Taipaleenmäki et al. (2013). MicroRNA functions in osteogenesis and dysfunctions in osteoporosis. Curr Osteoporos Rep 11:72-82.

20. Nelson TJ, A Martinez-Fernandez and A Terzic. (2010). Induced pluripotent stem cells: developmental biology to regenerative medicine. Nat Rev Cardiol 7:700-710.

21. Folmes CD, PP Dzeja, TJ Nelson and A Terzic. (2012). Metabolic plasticity in stem cell homeostasis and differentiation. Cell Stem Cell 11:596-606.

22. Naylor RM, DJ Baker and JM van Deursen. (2013). Senescent cells: a novel therapeutic target for aging and age-related diseases. Clin Pharmacol Ther 93:105-116 
23. Undale AH, JJ Westendorf, MJ Yaszemski and S Khosla. (2009). Mesenchymal stem cells for bone repair and metabolic bone diseases. Mayo Clin Proc 84:893-902.

24. Thatava T, YC Kudva, R Edukulla, K Squillace, JG De Lamo, YK Khan, T Sakuma, S Ohmine, A Terzic and Y Ikeda. (2013). Intrapatient variations in type 1 diabetes-specific iPS cell differentiation into insulin-producing cells. Mol Ther 21:228-239.

25. Higgins DM, R Wang, B Milligan, M Schroeder, B Carlson, J Pokorny, SH Cheshier, FB Meyer, IL Weissman, JN Sarkaria and JR Henley. (2013). Brain tumor stem cell multipotency correlates with nanog expression and extent of passaging in human glioblastoma xenografts. Oncotarget 4:792-801.

26. Smith AJ, NG Nelson, S Oommen, KA Hartjes, CD Folmes, A Terzic and TJ Nelson. (2012). Apoptotic susceptibility to DNA damage of pluripotent stem cells facilitates pharmacologic purging of teratoma risk. Stem Cells Transl Med 1:709-718.

27. Crespo-Diaz R, A Behfar, GW Butler, DJ Padley, MG Sarr, J Bartunek, AB Dietz and A Terzic. (2011). Platelet lysate consisting of a natural repair proteome supports human mesenchymal stem cell proliferation and chromo- somal stability. Cell Transplant 20:797811.

28. Behfar A, S Yamada, R Crespo-Diaz, JJ Nesbitt, LA Rowe, C Perez-Terzic, V Gaussin, C Homsy, J Bartunek and A Terzic. (2010). Guided cardiopoiesis enhances therapeutic benefit of bone marrow human mesenchymal stem cells in chronic myocardial infarction. J Am Coll Cardiol 56:721-734.

29. Psaltis PJ, KM Peterson, $\mathrm{R} \mathrm{Xu}$, F Franchi, T Witt, IY Chen, A Lerman, RD Simari, SS Gambhir and M Rodriguez-Porcel. (2013). Noninvasive monitoring of oxidative stress in transplanted mesenchymal stromal cells. JACC Cardiovasc Imaging 6:795-802.

30. Penfield JD, EC Gorospe and KK Wang. (2012). Tissue-engineered cell sheets for stricture prevention: a new connection between endoscopy and regenerative medicine. Gastroenterology 143:526-529.

31. Nelson TJ, A Behfar and A Terzic. (2008). Strategies for therapeutic repair: the $\mathrm{R}^{3}$ regenerative medicine paradigm. Clin Transl Sci 1:168-171.

32. Nelson TJ, A Behfar, S Yamada, A Martinez-Fernandez and A Terzic. (2009). Stem cell platforms for regenerative medicine. Clin Transl Sci 2:222-227.

33. Bartunek J, A Behfar, D Dolatabadi, M Vanderheyden, M Ostojic, J Dens,
B El Nakadi, M Banovic, B Beleslin et al. (2013). Cardiopoietic stem cell therapy in heart failure: the C-CURE (Cardiopoietic stem Cell therapy in heart failURE) multicenter randomized trial with lineage-specified biologics. J Am Coll Cardiol 61:2329-2338.

34. Faustino RS, DK Arrell, CD Folmes, A Terzic and C Perez-Terzic. (2013). Stem cell systems informatics for advanced clinical biodiagnostics: tracing molecular signatures from bench to bedside. Croat Med J 54:319-329.

35. Suresh R, A Chiriac, K Goel, HR Villarraga, F Lopez-Jimenez, RJ Thomas, A Terzic, TJ Nelson and C Perez-Terzic. (2013). CXCR4 $4^{+}$and FLK-1 ${ }^{+}$identify circulating cells associated with improved cardiac function in patients following myocardial infarction. J Cardiovasc Transl Res 6:787-797.

36. Terzic A, BS Edwards, KC McKee and TJ Nelson. (2011). Regenerative medicine: a reality of stem cell technology. Minn Med 94:44-47.

37. Nelson TJ and A Terzic. (2011). Induced pluripotent stem cells: an emerging theranostics platform. Clin Pharmacol Ther 89:648-650.

38. Waldman SA and A Terzic. (2012). Knowledge cycle transforms therapeutic innovation. Clin Pharmacol Ther 91:3-8. 